\title{
Pathological and imaging features of Paget's disease and nipple adenoma: a comparative study
}

\author{
Xuejing Liu, Yilin Xu, Junjun Liu, Shumeng Sun, Ying Zhu, Hong Lu \\ Department of Breast Imaging, Tianjin Medical University Cancer Institute and Hospital, National Clinical Research Center for Cancer, Key \\ Laboratory of Cancer Prevention and Therapy, Tianjin's Clinical Research Center for Cancer, Key Laboratory of Breast Cancer Prevention and \\ Therapy, Tianjin Medical University, Ministry of Education, Tianjin, China \\ Contributions: (I) Conception and design: X Liu, Y Xu; (II) Administrative support: H Lu; (III) Provision of study materials or patients: Y Zhu; (IV) \\ Collection and assembly of data: X Liu, S Sun; (V) Data analysis and interpretation: X Liu, J Liu; (VI) Manuscript writing: All authors; (VII) Final \\ approval of manuscript: All authors. \\ Correspondence to: Hong Lu, MD. Huan Hu Xi Road, Ti Yuan Bei, He Xi District, Tianjin 300060, China. Email: honglu@tmu.edu.cn.
}

Backgroundk Both Paget's disease (PD) and nipple adenoma (NA) are rare lesions occurred on nipple and share some similarities in clinical manifestations, but there are have different pathological manifestations and imaging findings. This study analyzed the clinicopathological and imaging features of PD in nipple and NA to improve our knowledge about these two diseases and to provide guidance for clinical diagnosis and treatment.

Methods: Retrospectively analyzed 99 female patients confirmed by surgery and pathology from January 2014 to December 2018. The features of imaging examination included 95 cases of breast ultrasound, 83 cases of breast X-ray and 24 cases of magnetic resonance imaging (MRI) were analyzed and compered the detection rate and diagnostic accuracy.

Results: The 99 patients consisted of 76 patients with PD and 23 patients with NA. Despite the similarity of clinical manifestations between PD and NA, the pathological features of these diseases were completely different. Differences in various imaging manifestations were found to facilitate differential diagnosis. Breast $\mathrm{X}$-ray and ultrasound can discover the nipple areola changes such as mass and calcification, but some cases still show negative. Breast MRI can clearly show the areola lesions of nipple PD and NA, accurately evaluate the degree and size of breast lesions, and help clinicians choose appropriate and personalized diagnosis and treatment methods.

Conclusions: The combination of multiple breast imaging examinations (including X-ray, ultrasound, and MRI) can improve the diagnosis of PD and NA and play a guiding role in the options for clinical treatment.

Keywords: Paget's disease (PD); nipple adenoma (NA); X-ray; ultrasonography; magnetic resonance imaging (MRI)

Submitted Nov 25, 2021. Accepted for publication Jan 07, 2022.

doi: $10.21037 / g s-21-862$

View this article at: https://dx.doi.org/10.21037/gs-21-862

\section{Introduction}

Paget's disease (PD) of the nipple is a rare breast malignant disease caused by the infiltration of epidermoid carcinoma cells into the nipple and/or areola. PD accounts for 3\% of female breast malignant tumors. Nipple adenoma (NA) is even rarer, with an incidence of less than $1 \%$ in benign breast tumors. Both PD and NA occurred in the nipple and share some similarities in clinical manifestations, such as enlargement, rupture, and repeated scab of the nipple. Therefore, it is difficult to accurately distinguish between PD and NA. However, PD and NA have different pathological manifestations and imaging findings. Previous reports on PD and NA were mostly case reports or 
pathological differential diagnosis, and imaging reports were rare. Because the surgical methods and prognosis of the two diseases are different, patients can benefit greatly if they can make differential diagnosis of the two diseases in the imaging examination before operation (1-3). In this study, we analyzed the clinical and imaging features, including breast ultrasound, $\mathrm{X}$-ray, and magnetic resonance image (MRI), of PD of the nipple and NA to investigate the main points of differential diagnosis and to provide an accurate basis for clinical preoperative diagnosis. We present the following article in accordance with the STARD reporting checklist (available at https://gs.amegroups.com/ article/view/10.21037/gs-21-862/rc).

\section{Methods}

A total of 99 female patients with nipple PD $(n=76)$ and NA $(\mathrm{n}=23)$, which were confirmed by surgery and pathology in Tianjin Medical University Cancer Institute and Hospital from January 2014 to December 2018, were analyzed retrospectively. All procedures performed in this study involving human participants were in accordance with the Declaration of Helsinki (as revised in 2013). The study was approved by institutional ethics board of Tianjin Medical University Cancer Institute and Hospital (No. bc2018015). Individual consent for this retrospective analysis was waived. All patients underwent imaging examinations before surgery. Among them, 95 patients received a breast ultrasound, 83 patients received a breast X-ray, and 24 patients received MRI scans. Patients were excluded if they satisfied the following criteria: (I) the patient underwent neoadjuvant therapy before the imaging examination; and (II) the patient had incomplete imaging data or the data was of poor image quality.

\section{Breast $X$-ray}

Selenia full-digital breast $\mathrm{X}$-ray radiographic equipment (Hologic, United States) was used for the breast X-ray examination, accompanied by a matching imaging workstation for image observation. Photography used a fully automatic exposure system. The patients routinely received breast $\mathrm{X}$-ray examinations in craniocaudal position and mediolateral oblique position, as well as spot compression and magnification mammography if necessary.

\section{Breast ultrasound}

Logiq E9 color Doppler ultrasound (GE company, United States) was used to perform the breast ultrasound examination, with the frequency of the linear probe of 6-15 MHz. The patients were routinely placed in supine or lateral positions to fully expose the breast. During the examination, the breast was scanned continuously in cross section and vertical section and was radially scanned while centered on the nipple. The examination sections covered each other to avoid omission, especially of the gland margin and the nipple areola. The morphological and hemodynamic characteristics of the lesions were observed comprehensively.

\section{Breast MRI}

The 3.0T MRI scanner (GE company, United States) was used to perform breast MRI, with the 8-channel breast phased-array surface coil pairs. The patients were placed in a prone position with both breasts naturally prolapsed to complete the bilateral breast plain scan and dynamic contrast-enhanced MRI. The scan parameters and sequence were as follows: (I) axial fast spin echo T1 weighted image repetition time (TR): $622 \mathrm{~ms}$; echo time (TE): $10 \mathrm{~ms}$; (II) fat suppression T2 weighted image scanning in axial view and sagittal view of the breast (TR: $6,330 \mathrm{~ms}$; TE: $68 \mathrm{~ms}$ ) was performed, with the slice thickness of $4.5 \mathrm{~mm}$, a slice gap of $0.5 \mathrm{~mm}$, the matrix of $384 \times 224$, and the number of excitations (NEX) of 2; (III) sagittal dynamic contrast enhanced magnetic resonance imaging (MRI) was performed using the 3D Fastcard gradient echo train and volume imaging for breast assessment (VIBRANT). The mask was scanned before dynamic enhancement, and then the contrast gadopentetic acid dimeglumine salt injection (Bayer) was injected through the dorsal vein of the hand with a high-pressure injector at a dose of $0.2 \mathrm{~mL} / \mathrm{kg}$ and a rate of $2.0 \mathrm{~mL} / \mathrm{s}$. At the same time, the same amount of normal saline was injected. Five consecutive phases were scanned immediately for a time of up to 8 minutes. The scanning parameters were as follows: TR was $6.1 \mathrm{~ms}$, TE was $2.9 \mathrm{~ms}$, the flip angle was $15^{\circ}$, the matrix was $256 \times 128$, slice thickness was $1.8 \mathrm{~mm}$, and NEX was 1 ; and (IV) the delayed axial VIBRANT sequence was scanned.

\section{Image interpretation}

The images of breast ultrasounds, X-rays, and MRIs 
Table 1 Clinical manifestations of PD of the nipple $(n=76)$ and NA $(n=23)$

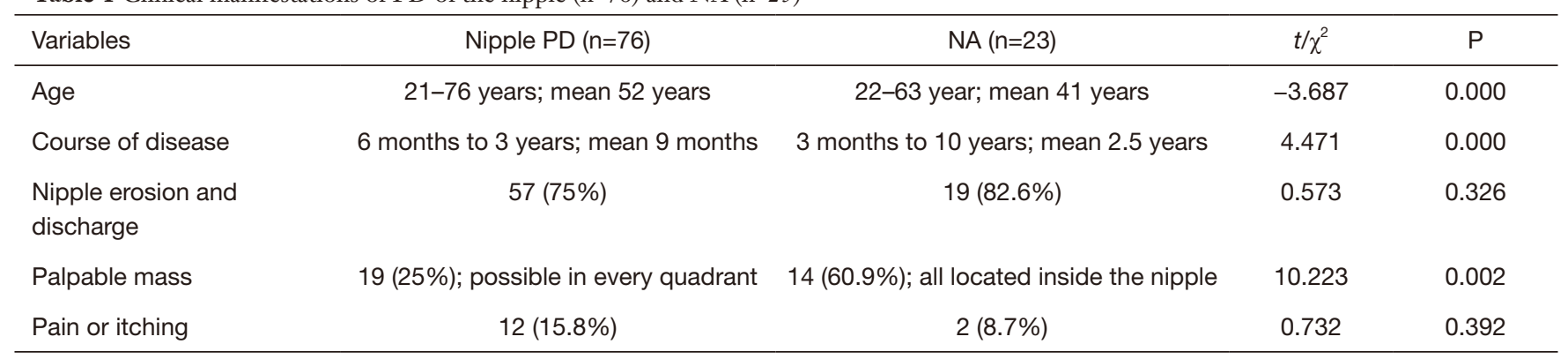

PD, Paget's disease; NA, nipple adenoma.

were read and analyzed by three breast radiologists (two 5 -year experience radiologists and one 10-year experience radiologists) separated. Analysis and diagnosis were done by referring to the diagnostic criteria from the Breast Imaging and Reporting Data System Ultrasound (BI-RADS) proposed by the American College of Radiology. In this study, BI-RADS 4B or above was diagnosed as malignant, and BI-RADS $4 \mathrm{~A}$ or below was diagnosed as benign. The detection rate and diagnostic accuracy of the three imaging examinations were analyzed.

\section{Statistical analysis}

SPSS 22.0 (IBM Corp, Armonk, NY, USA) was used to perform the statistical analysis. Counting data were expressed by frequency and tested by $\chi^{2}$, Fisher $t$-test was used for measurement data, and $\mathrm{P}<0.05$ was considered statistically significant.

\section{Results}

All cases were confirmed by surgery and pathology, including 76 patients with nipple PD and 23 patients with NA. Of the 76 patients with nipple PD, 4 were simple PD, 23 were PD with ductal carcinoma in situ (DCIS), 6 were $\mathrm{PD}$ with microinvasive carcinoma, 2 were $\mathrm{PD}$ with invasive micropapillary carcinoma, 2 were PD with invasive lobular carcinoma, and 39 were PD with invasive ductal carcinoma. All 23 cases with NA were benign without cancerization. The comparison of clinical manifestations between PD of the nipple and NA is shown in Table 1.

\section{Breast $X$-ray features}

A total of 83 patients with breast diseases received X-ray imaging, including 65 patients with PD of the nipple and 18 patients with NA. In the PD of the nipple group, a breast $\mathrm{X}$-ray diagnosed 53 patients accurately, detected 63 cases, and presented negative results in 2 cases. In the NA group, 15 patients had an accurate diagnosis, 9 were detected, and 9 were negative. PD can be characterized by thickening, flattening, and calcification of the nipple, but the X-ray findings showed no significant difference between these factors in patients with NA and PD. However, PD was usually accompanied by malignant lesions in other parts of the breast, while NA was mainly characterized by breast nodules or the local density of the nipple areola, and there was a significant difference in the $\mathrm{X}$-ray detection rate between the two groups $(\mathrm{P}<0.05$; Table 2).

\section{Features of the breast ultrasound}

A total of 95 patients with breast diseases were examined with a breast ultrasound, including 73 patients with $\mathrm{PD}$ of the nipple and 22 patients with NA. In the PD of the nipple group, a breast ultrasound diagnosed 58 patients accurately, detected 69 cases, and presented negative results in 4 cases. In the NA group, 19 patients had an accurate diagnosis, and 22 cases were detected. PD had a variety of ultrasonographic manifestations, such as nipple enlargement, intranipple duct dilatation, and calcification, but these were not statistically significant in our study compared with the nipple changes of the papillary adenoma. Only abundant blood flow in the nipple and the malignant lesions in other parts of the breast had statistical significance in patients with $\mathrm{PD}$ $(\mathrm{P}<0.05)$. Patients with NA only showed nipple changes, that is, intranipple nodules and breast lesions. There was poor blood flow in the nipple and no malignant lesions in other parts, and the difference was statistically significant in patients with $\mathrm{NA}(\mathrm{P}<0.05$; Table 3). 
Table 2 X-ray manifestations of 83 patients with breast diseases (65 patients with PD of the nipple and 18 patients with NA)

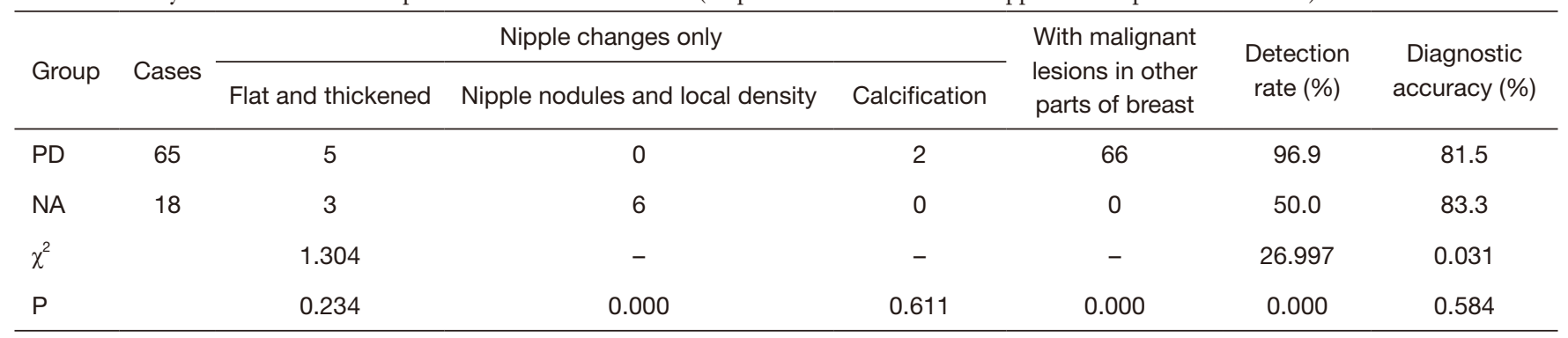

PD, Paget's disease; NA, nipple adenoma; -, Fisher exact probability method showed no statistical value.

Table 3 Ultrasound manifestations of 95 patients with breast lesions ( 73 patients with PD of the nipple and 22 patients with NA)

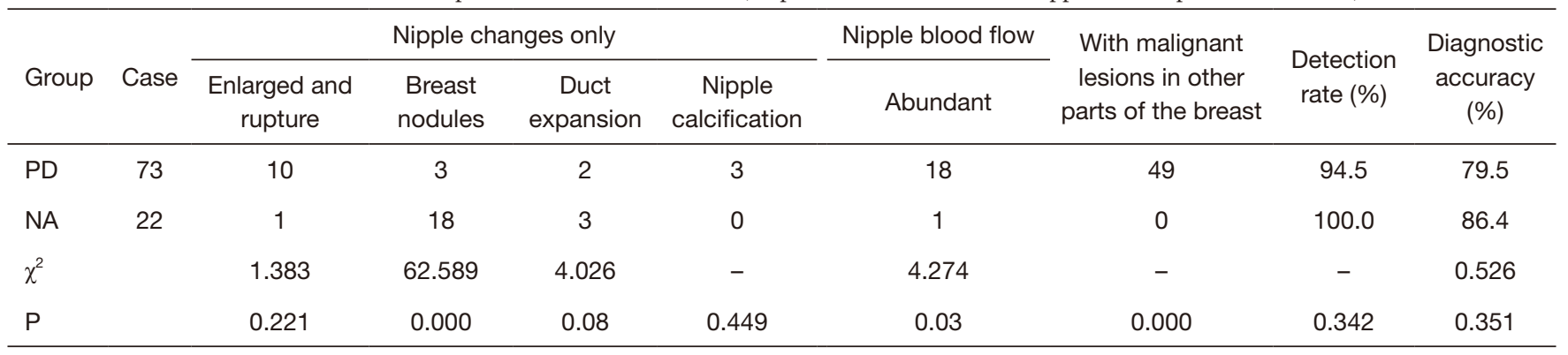

PD, Paget's disease; Na, Nipple adenoma; -, Fisher exact probability method, no statistical value.

\section{Features of breast MRI}

A total of 24 patients with breast diseases were examined using breast MRI, including 19 patients with PD of the nipple and 5 patients with NA. In the PD of the nipple group, the breast MRI diagnosed 19 cases accurately and detected 19 cases. While in the NA group, 4 cases had an accurate diagnosis, and 5 were detected. As for patients with $\mathrm{PD}$ of the nipple, the mass lesion had irregular morphology and a fuzzy margin, while the non-mass lesion was mainly enhanced along the duct and segment. As for patients with NA, the mass lesions were mainly characterized by breast nodules that had an oval shape and a smooth margin, while the non-mass lesions only showed enhancement in the nipple areola. There was a significant difference between the $\mathrm{PD}$ and $\mathrm{NA}(\mathrm{P}<0.05 ;$ Tables 4,5$)$.

\section{Discussion}

There are many subtypes of breast nipple lesions, including benign lesions (such as NA, papillary inflammation, and ductal papilloma) and malignant lesions (such as PD of the nipple, nipple lymphoma, and invasive ductal carcinoma) (4). Some lesions inside the breast can also involve the nipple (2). Healthy women have symmetrical sized and morphologically bilateral nipples without retraction and depression. Patients with PD of the nipple and NA share similar clinical manifestations, such as nipple enlargement, discharge, scabbing, swelling, and erosion, among others. However, the nature of the two diseases, and treatment for them, is completely different $(3,5,6)$. Therefore, a correct preoperative diagnosis is extremely critical to the operation plan and prognosis.

$\mathrm{PD}$ of the breast is a rare malignant lesion that occurs in people in a wide range of ages, but mostly presents in women aged between 40 and 60 years old. It is characterized by the appearance of malignant glandular epithelial cells in the nipple and/or areola epidermis. Clinically, PD commonly presents as redness, eczema, exudation, and scabbing. Reports have shown that more than $90 \%$ of patients also have malignant lesions in other parts of the breast, which can be DCIS or a combination of DCIS and invasive carcinoma $(7,8)$. NA is a relatively rare benign lesion in the nipple and is also known as florid papillomatosis, florid adenomatosis, intraductal papillomatosis under the areola, and invasive adenomatosis. It occurs in women of all ages and can present as nipple pain, swelling, ulcers, and nipple discharge, and 
Table 4 MRI manifestations of breast mass lesions

\begin{tabular}{|c|c|c|c|c|c|c|c|}
\hline \multirow{2}{*}{ Group } & \multirow{2}{*}{ Cases } & \multicolumn{6}{|c|}{ Mass lesion } \\
\hline & & Oval & Irregular & Smooth & Fuzzy & Even & Uneven \\
\hline PD & 19 & 0 & 6 & 0 & 6 & 0 & 6 \\
\hline NA & 5 & 2 & 0 & 2 & 0 & 1 & 1 \\
\hline $\mathrm{P}$ & & \multicolumn{2}{|c|}{0.036} & \multicolumn{2}{|c|}{0.036} & \multicolumn{2}{|c|}{0.25} \\
\hline
\end{tabular}

PD, Paget's disease; NA, nipple adenoma; -, Fisher exact probability method, no statistical value.

Table 5 MRI manifestations of non-mass-like breast lesions

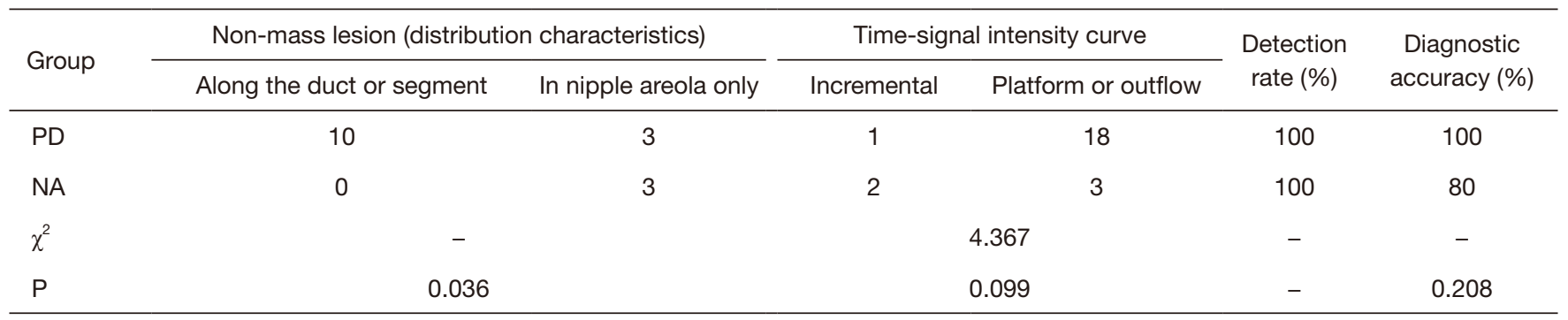

PD, Paget's disease; NA, nipple adenoma; -, Fisher exact probability method, no statistical value.

thus is difficult to distinguish from PD. The features of NA are benign hyperplasia of glands and ducts within the fibrous stroma of varying sizes, with well-defined boundaries. The glands can be relatively simple and adenopathy-like or distorted and dilated due to significant papillary hyperplasia and/or common ductal hyperplasia, and necrotic foci can be observed, as well as large sudoriferous glands or squamous metaplasia (9-11). In this study, although PD of the nipple and NA showed similar clinical manifestations, it is not difficult to make diagnosis by doing cytological examination. PD is characterized by the presence of Paget cells under the microscope. The cells are located in the papillary epidermis. They are light stained large, round and oval cells, containing mucus, scattered or arranged in nests. The cytoplasm is rich and transparent, with obvious nucleoli, irregular and heterosexual nuclei. The epidermis can have hyperplasia and dyskeratosis. Although periglandular interstitial sclerosis or papillary ductal epithelial hyperplasia can squeeze, distort and form the shape of pseudoinfiltration, the cell size and shape of NA are relatively consistent, the mitotic image is rare, and the myoepithelium in the outer layer is complete, which is also the key point of differentiation from PD. They also had certain differences in patients' ages, the course of the disease, and nipple manifestations. Compared with patients with NA, those with PD of the nipple were of an older average age, had a shorter average course of the disease, and often occur nipple erosion and discharge. PD of the nipple mainly shows bloody exudation and is often accompanied by itching and a mass inside the breast. Patients with NA have similar nipple manifestations, but the exudation is clearer and less accompanied by itching, and the mass is confined to the nipple.

Breast X-rays showed abnormalities in the nipple and areola and under the areola, including skin thickening, nipple retraction, malignant calcification, and areola masses. Other breast lesions were also observed, including masses with suspicious signs, asymmetric density, structural distortion, or malignant calcification (Figures 1,2). A total of 18 patients with NA underwent an X-ray examination in our study, and half (9/18) showed negative, while the other patients only showed nipple changes, nipple enlargement, nodules, or the focal asymmetry of nipple areola, which was in line findings in the diagnosis that showed these tumors were benign. A total of 65 patients with PD underwent X-ray examination, of which $86.2 \%(56 / 65)$ showed positive including nipple retraction, malignant calcification, and 
areola masses, which was consistent with most reports. $\mathrm{X}$-ray is helpful for distinguish between PD and NA. PD usually can find calcification in nipple and malignant mass or calcification in the breast, NA only showed the change of nipple. However, literature showed that $22 \%$ to $50 \%$ of patients had negative X-ray manifestations. Other reports showed that nearly $50 \%$ of patients with typical clinical PD signs showed negative breast $\mathrm{X}$-ray manifestations (12-14). Furthermore, the breast $\mathrm{X}$-ray imaging method has some
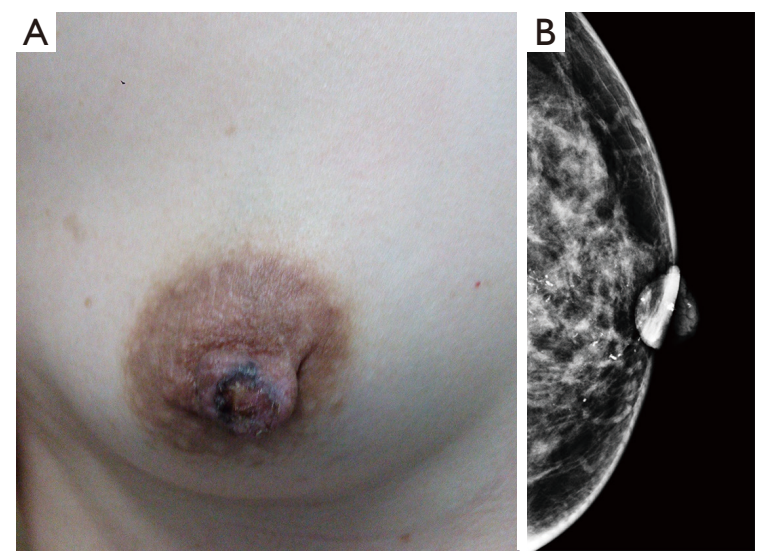

Figure 1 The patient was a 45 -year-old female with papillary eczema that had been recurrent for 2 years. (A) The X-ray showed diffuse pleomorphic calcification in the left breast and thickening of the skin layer in the areola area; (B) Paget's disease combined with invasive ductal carcinoma was pathologically confirmed. limitations in the diagnosis of lesion size. For example, $43 \%$ of lesions turned out to be larger than the lesion range shown by a breast X-ray, therefore a breast ultrasound or MRI examination should also be considered (12).

Ultrasound imaging is particularly important for patients with negative breast $\mathrm{X}$-ray findings because it can discover breast lesions in these patients. Ultrasonographic features of PD include masses, microcalcification, ductal dilatation, and morphological changes in the areola (such as flattening, asymmetry, and thickening) (15). In the late stage, masses can be detected in other parts of the gland. In our study, most patients (54/73) had lesions in parts of the breast other than the nipple, which was consistent with the reports in the literature. Due to the low incidence of NA, literature about its ultrasonographic findings only includes case reports. Ultrasonographic findings can be described as hypoechoic nodules with a clear inner boundary, enhanced posterior echo, and uniform internal echo, which are round or oval in shape, and which have a small amount of blood flow or marginal blood flow inside $(15,16)$. In our study, 22 cases of NA were examined by ultrasound. All the lesions were limited to the nipple, and most of the patients (18/22) showed intrapapillary nodules. The main points of ultrasonographic differentiation between $\mathrm{PD}$ and NA are that fewer patients with $\mathrm{PD}$ of the nipple present nipple changes alone, otherwise, they mainly manifest as nipple enlargement and rupture, and the color Doppler shows significantly increased nipple blood flow signals on the
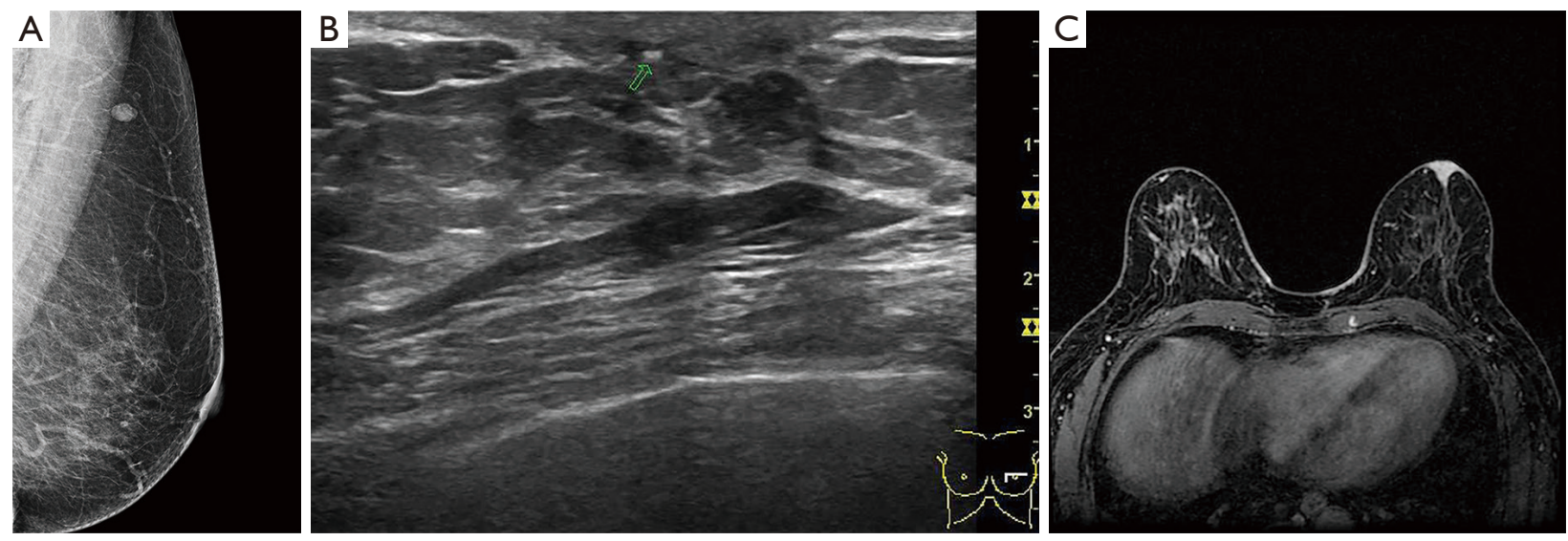

Figure 2 The patient was a 62-year-old female with nipple erosion that had been present for 1 year. MLO of the left breast showed that the skin layer of the left nipple and areola was thickened, and the axillary lymph nodes were enlarged. (A) The ultrasound showed that the nipple was enlarged, and the strong echo light spot was behind the nipple; (B) breast magnetic resonance imaging showed abnormal enhancement of the nipple areola area; (C) Paget's disease combined with ductal carcinoma in situ was confirmed by pathology. MLO, mediolateral oblique. 
trouble side rather than on the health side. Lesions in other parts of the breast can be found in most patients; but NA often has hypoechoic nodules inside the nipple, with clear boundaries, even echoes, and rare malignant lesions.

However, not all breast lesions can be detected by ultrasound. Some studies have shown that $13 \%$ of patients with PD cannot be detected by ultrasound or X-ray (16). MRI plays an important role in the preoperative examination of nipple areola lesions, especially in patients with negative breast $\mathrm{X}$-ray and ultrasound results. Breast MRI can accurately show abnormal nipple enhancement, nipple areola thickening and enhancement, evaluate the lesion extent, and exclude DCIS or invasive carcinoma (Figure 2). In contrast-enhanced images, a normal nipple enhances in various ways, such as showing no enhancement, mild enhancement, or obvious enhancement, however, the nipple in all instances is bilaterally symmetrical. Compared with the normal side, the nipple or areola of the abnormal side can show asymmetric, nodular, diskshaped, or irregular enhancement. MRI images are highly sensitive during diagnosis and evaluation of nipple tumors and tumors near the nipple. In this study, MRI examination showed the lesions in the nipple areola and inside the breast among patients with PD of the nipple with a diagnostic accuracy of up to $100 \%$. Although the features of MRI images in PD are not specific to those of DCIS or an invasive carcinoma inside the breast, they can clarify the extent of the lesion and help formulate a reasonable treatment. Some literature explored the MRI findings of NA, which may be characterized by an enlarged nipple with enhanced nodules in the nipple (17-19). In this study, NA presented as mass or non-mass lesions, which were all restricted to the nipple areola without other lesions. PD of the nipple has completely different surgical options than the options for NA. PD of the nipple requires excision of the nipple areola and other malignant lesions in the breast, and postoperative radiotherapy or endocrine therapy should be continued according to the pathological. However, NA generally needs to remove the local lesions, including the nipple areola lesions only, and there is usually no recurrence after surgery (20). Therefore, MRI has a high sensitivity and accuracy and can effectively display and evaluate nipple areola lesions, suspicious lesions in other parts of the breast, and multifocal and multicentric lesions (21). MRI can also determine the nature and extent of surgery that will be needed and can be used as a tool for follow-up and reexamination after surgery.

Our study has some limitations. (I) This retrospective study evaluated ultrasound images based on the images stored by ultrasound physicians, which lacked the comprehensiveness of lesions. (II) In this study, NA is a kind of rare disease. In this study, the number of patients with breast MRI before operation and confirmed by pathology is small. Some patients who underwent MRI but did not undergo surgery were excluded from this study, leading to the resulting of detection rate and diagnostic accuracy of MRI are not representative. (III) This study is a retrospective analysis, which is likely to cause some deviations in the results. It needs to be further confirmed by multi-center clinical trials.

\section{Conclusions}

Although PD of the nipple and NA have similar clinical manifestations, their pathological and imaging findings have different characteristics. The average age of nipple $\mathrm{PD}$ is older, the average course of disease is shorter, and the nipple changes are more serious. The nipple exudate of NA patients is clear, and the mass is limited to the nipple. Most cases with $\mathrm{PD}$ of the nipple are accompanied by malignant lesions in other areas of the breast, therefore it is very important to accurately evaluate the location and extent of these lesions using preoperative imaging examination. Although PD in X-ray and ultrasound can observe nipple enlarged and flat, with malignant calcification or mass in the breast, while NA usually only showed nipple enlarged or mass in the nipple, some patients still have negative breast $\mathrm{X}$-ray or ultrasound findings. Breast MRI is an effective tool for preoperative evaluation of breast diseases. It can clearly exhibit the areola lesions in PD of the nipple and $\mathrm{NA}$, as well as malignant lesions in other areas of the breast. Moreover, breast MRI can accurately evaluate the extent of the breast lesion, which helps clinicians choose appropriate and personalized diagnoses and treatments.

\section{Acknowledgments}

Funding: This study was supported by the Chinese National Key Research and Development Project (Grant number 2018YFC1315601) and Tianjin Health Research Project (Grant number KJ20024).

\section{Footnote}

Reporting Checklist: The authors have completed the STARD reporting checklist. Available at https://gs.amegroups.com/ 
article/view/10.21037/gs-21-862/rc

Data Sharing Statement: Available at https://gs.amegroups. com/article/view/10.21037/gs-21-862/dss

Conflicts of Interest: All authors have completed the ICMJE uniform disclosure form (available at https://gs.amegroups. com/article/view/10.21037/gs-21-862/coif). The authors have no conflicts of interest to declare.

Ethical Statement: The authors are accountable for all aspects of the work in ensuring that questions related to the accuracy or integrity of any part of the work are appropriately investigated and resolved. All procedures performed in this study involving human participants were in accordance with the Declaration of Helsinki (as revised in 2013). The study was approved by institutional ethics board of Tianjin Medical University Cancer Institute and Hospital (No. bc2018015). Individual consent for this retrospective analysis was waived.

Open Access Statement: This is an Open Access article distributed in accordance with the Creative Commons Attribution-NonCommercial-NoDerivs 4.0 International License (CC BY-NC-ND 4.0), which permits the noncommercial replication and distribution of the article with the strict proviso that no changes or edits are made and the original work is properly cited (including links to both the formal publication through the relevant DOI and the license). See: https://creativecommons.org/licenses/by-nc-nd/4.0/.

\section{References}

1. Elbendary A, Xue R, Valdebran M, et al. Diagnostic Criteria in Intraepithelial Pagetoid Neoplasms: A Histopathologic Study and Evaluation of Select Features in Paget Disease, Bowen Disease, and Melanoma In Situ. Am J Dermatopathol 2017;39:419-27.

2. Sanders MA, Brock JE, Harrison BT, et al. Nipple-invasive primary carcinomas: clinical, imaging, and pathologic features of breast carcinomas originating in the nipple. Arch Pathol Lab Med 2018;142:598-605.

3. Xu W, Chen S, Zhou X, et al. Nipple adenoma of breast: 4 cases report and literature review. J Clin Exp Pathol 2019;35:1082-5.

4. Lakhani SR, Ellis IO, Schnitt SJ. WHO classification of tumours of the breast. IARC: Lyon, 2012:149-52.

5. Hu XW, Liu H. Advances in clinicopathological and genetic studies of mammary Paget's disease. Zhonghua Bing Li Xue Za Zhi 2020;49:653-7

6. Spohn GP, Trotter SC, Tozbikian G, et al. Nipple adenoma in a female patient presenting with persistent erythema of the right nipple skin: case report, review of the literature, clinical implications, and relevancy to health care providers who evaluate and treat patients with dermatologic conditions of the breast skin. BMC Dermatol 2016;16:4.

7. Wang J, Yu WJ, Wang X, et al. Clinicopathological characteristics and prognosis analysis with Paget's disease of the breast. Zhonghua Zhong Liu Za Zhi 2018;40:523-7.

8. Kothari AS, Beechey-Newman N, Hamed H, et al. Paget disease of the nipple: a multifocal manifestation of higherrisk disease. Cancer 2002;95:1-7.

9. Wang J, Tai Y, Zhang H, et al. Nipple adenoma of the breast: Clinicopathologic analyses of 5 cases. Chin J Diagnostic Pathol 2020;27:609-13.

10. Liau JY, Lee YH, Tsai JH, et al. Frequent PIK3CA activating mutations in nipple adenomas. Histopathology 2017;70:195-202.

11. Barco I, Vidal M, Fraile M, et al. MOHS micrographic surgery for treating erosive adenoma of the nipple: a case report and review of the literature. Int J Dermatol 2017;56:1451-4.

12. Shi J, Zhu T, Zhang T, et al. Imaging Discovery and Pathological Analysis of Paget's Disease of Breast. J Clin Radiol 2018;37:219-22.

13. Lim HS, Jeong SJ, Lee JS, et al. Paget disease of the breast: mammographic, US, and MR imaging findings with pathologic correlation. Radiographics 2011;31:1973-87.

14. Günhan-Bilgen I, Oktay A. Paget's disease of the breast: clinical, mammographic, sonographic and pathologic findings in 52 cases. Eur J Radiol 2006;60:256-63.

15. Wei $Y, Z$ Zhu Q, Li J, et al. Clinical and Sonographic Features of Mammary Paget's Disease. Zhongguo Yi Xue Ke Xue Yuan Xue Bao 2017;39:396-400.

16. Chiorean A, Pintican RM, Szep M, et al. Nipple Ultrasound: A Pictorial Essay. Korean J Radiol 2020;21:955-66.

17. Sun D, Li J, Zhang L. A case report and literature review of Nipple adenoma. Chin J Breast Dis(Electronic Edition) 2016;10:370-2.

18. Chen S, Chen H, Yi Y, et al. Comparative study of breast cancer with or without concomitant Paget disease: An analysis of the SEER database. Cancer Med 2019;8:4043-54. 
19. Zhao Y, Wang L, Luo R, et al. The MR imaging features of breast nipple disease. Radiol Practice 2015 30:1085-8.

20. Fujisawa K, Kato M, Kono T, et al. Nipple adenoma in a 2-year old boy. Pediatr Dermatol 2018;35:e184-e185.

21. Yao Y, Sun L, Meng Y, et al. Breast-Conserving surgery

Cite this article as: Liu X, Xu Y, Liu J, Sun S, Zhu Y, Lu H. Pathological and imaging features of Paget's disease and nipple adenoma: a comparative study. Gland Surg 2022;11(1):207-215. doi: $10.21037 / g s-21-862$ in patients with mammary Paget's disease. J Surg Res 2019;241:178-87.

(English Language Editor: C. Mullens) 